\title{
Improving Adherence to Smoking Cessation Treatment: Intervention Effects in a Web-Based Randomized Trial
}

\author{
Amanda L. Graham PhD ${ }^{1,2}$, George D. Papandonatos PhD ${ }^{3}$, \\ Sarah Cha MSPH ${ }^{1}$, Bahar Erar MS ${ }^{3}$, Michael S. Amato PhD', \\ Nathan K. Cobb PhD, ${ }^{4,5}$, Raymond S. Niaura PhD ${ }^{1,2,6}$, \\ David B. Abrams PhD 1,2,6
}

'Schroeder Institute for Tobacco Research and Policy Studies, Truth Initiative, Washington, DC; ${ }^{2 D e p a r t m e n t ~ o f ~}$ Oncology, Georgetown University Medical Center/Cancer Prevention and Control Program, Lombardi Comprehensive Cancer Center, Washington, DC; ${ }^{3}$ Center for Statistical Science, Brown University, Providence, Rl; ${ }^{4}$ MeYouHealth, Boston, MA; ${ }^{5}$ Department of Pulmonary and Critical Care, Georgetown University Medical Center, Washington, DC; ${ }^{6}$ Department of Health, Behavior and Society, The Johns Hopkins Bloomberg School of Public Health, Baltimore, MD

Corresponding Author: Amanda L. Graham, PhD, Schroeder Institute for Tobacco Research and Policy Studies, Truth Initiative, 900 G Street NW, Fourth Floor, Washington, DC 20001, USA. Telephone: 202-454-5938; Fax: 202-454-5785; E-mail: agraham@truthinitiative.org

\begin{abstract}
Background: Web-based smoking cessation interventions can deliver evidence-based treatments to a wide swath of the population, but effectiveness is often limited by insufficient adherence to proven treatment components. This study evaluated the impact of a social network (SN) intervention and free nicotine replacement therapy (NRT) on adherence to evidence-based components of smoking cessation treatment in the context of a Web-based intervention.

Methods: A sample of adult U.S. smokers $(N=5290)$ was recruited via BecomeAnEX.org, a free smoking cessation Web site. Smokers were randomized to one of four arms: (1) an interactive, evidence-based smoking cessation Web site (WEB) alone; (2) WEB in conjunction with an SN intervention designed to integrate participants into the online community (WEB+SN); (3) WEB plus free NRT (WEB+NRT); and (4) the combination of all treatments (WEB+SN+NRT). Adherence outcomes assessed at 3-month follow-up were as follows: Web site utilization metrics, use of skills training components, intratreatment social support, and pharmacotherapy use.

Results: WEB+SN+NRT outperformed all others on Web site utilization metrics, use of practical counseling tools, intratreatment social support, and NRT use. It was the only intervention to promote the sending of private messages and the viewing of community pages overWEB alone. Both social network arms outperformed WEB on most metrics of online community engagement. Both NRT arms showed higher medication use compared to WEB alone.

Conclusions: This study demonstrated the effectiveness of two approaches for improving adherence to evidence-based components of smoking cessation treatment. Integrated approaches to medication provision and social network engagement can enhance adherence to components known to improve cessation.

Implications: This study demonstrated that an integrated approach to medication provision and social network integration, when delivered through an online program, can enhance adherence
\end{abstract}


across all three recommended components of an evidence-based smoking cessation program (skills training, social support, and pharmacotherapy use). Nicotine replacement therapy-when provided as part of an integrated program-increases adherence to other program elements, which in turn augment its own therapeutic effects. An explicit focus on approaches to improve treatment adherence is an important first step to identifying leverage points for optimizing intervention effectiveness.

\section{Introduction}

Web-based smoking cessation interventions can help reduce the population prevalence of smoking. They are well suited to deliver the core components of evidence-based cessation treatment recommended in national treatment guidelines ${ }^{1}$ : (1) Skills training information and feedback can be tailored and produced immediately; (2) Social support from peers and experts can be provided in online communities; and (3) Information about pharmacotherapy and medication can be delivered efficiently through automated systems, including tailored guidance for medication selection and dosing. In addition, Web-based interventions can reach and engage large numbers of smokers given their accessibility, scalability, and cost effectiveness. $^{2-4}$ Their effectiveness has been demonstrated in numerous systematic reviews and meta-analyses, ${ }^{5-8}$ yet the mechanisms by which Web-based interventions promote abstinence remain poorly understood. The majority of studies conducted have focused on the comparative effectiveness of Web-based interventions, ${ }^{5}$ with few trials designed specifically to test hypotheses about mechanisms of action. ${ }^{9-12}$ Moving beyond a singular focus on outcomes to understand how and why interventions influence abstinence-the core focus of implementation science ${ }^{13-15}$ - will enable faster and more efficient optimization of Web-based interventions. ${ }^{10,16}$

Adherence to an intervention is a primary determinant of the effectiveness of smoking cessation treatment. ${ }^{1}$ For Web-based interventions, adherence is typically measured by overall Web site utilization metrics (eg, Web site logins, page views, and features used). ${ }^{17-19}$ Observational studies have confirmed that greater utilization of Web-based cessation interventions is associated with a higher probability of abstinence..$^{20-25}$ Only a few experimental studies have attempted to directly influence abstinence by promoting engagement, and results have been mixed. Strecher and colleagues ${ }^{12}$ and McClure and colleagues ${ }^{10,26}$ examined the impact of specific Internet program features on engagement and abstinence. Strecher tested personalized message source, tailored outcome expectations, efficacy expectations, success story messages, and either fully available or sequentially available program content. McClure tested message tone, navigation autonomy, E-mail reminders, and receipt of personally tailored testimonials. Both trials demonstrated increases in engagement with the program elements that were tested, but only Strecher found that higher levels of engagement translated to increased abstinence rates.

Building on the work of Strecher, ${ }^{12}$ McClure ${ }^{10,26}$, and others, ${ }^{27,28}$ this study was a pragmatic randomized trial ${ }^{29}$ specifically designed to advance implementation science by examining how different interventions drive adherence to the core components of evidence-based cessation treatment (skills training, social support, and pharmacotherapy). The first intervention drew upon Social Cognitive Theory and evidence that observation, modeling, and adjustment to community norms are powerful influences on behavior ${ }^{30-32}$ and engagement with online interventions. ${ }^{28}$ We used a social network approach designed to increase the proportion of smokers who participate in and become integrated into an existing online social network. We hypothesized that increasing an individual's social ties and exposure to social influence would encourage ongoing community engagement, accelerate information seeking, and prompt and reinforce use of nicotine replacement therapy (NRT) and skills training tools. The second intervention provided free access to NRT. Removing initial cost and access barriers can introduce pharmacotherapy to smokers who might not otherwise try it and induce a quit attempt. ${ }^{33}$ Covering the cost of NRT may also result in greater adherence to recommended dosage and duration of use,,$^{34}$ as well as greater engagement with the Web site and community as an ancillary benefit of information seeking regarding NRT.

Thus, the overall aim of this study was to evaluate the comparative effectiveness of a social network (SN) intervention and free NRTalone and in combination-on increasing adherence to the three evidence-based components of smoking cessation treatment. ${ }^{1}$ The primary outcome of interest was treatment adherence, defined and measured as (a) overall treatment "dose" received (general Web site utilization metrics), (b) use of skills training treatment components (interaction with static and interactive features on the Web site), (c) exposure to intratreatment social support (engagement in the online community), and (d) use of pharmacotherapy. We hypothesized that both the SN and NRT interventions would increase treatment adherence and that the combined intervention would yield larger increases in adherence than either intervention alone. Grounded in an implementation science framework, ${ }^{13,14,35}$ this paper is purposefully focused on understanding how interventions enhance treatment adherence as a necessary and sufficiently complex first step toward improving treatment effectiveness. The impact of enhanced adherence on smoking cessation outcomes will be reported in a separate manuscript.

\section{Methods}

\section{Study Design}

The full study protocol has been published elsewhere. ${ }^{36}$ Briefly, this study compared the effectiveness of an evidence-based smoking cessation Web site (WEB) alone and in conjunction with (1) a theory-driven, empirically informed social network ( $\mathrm{SN}$ ) intervention designed to integrate participants into the online community and (2) access to an initial course of free NRT. Using a $2(\mathrm{SN}$, no $\mathrm{SN}) \times 2$ (free NRT, no NRT) randomized, controlled factorial design with repeated measures at baseline and at 3 and 9 months, participants were randomized to $\mathrm{WEB}, \mathrm{WEB}+\mathrm{SN}$, WEB+NRT, or $\mathrm{WEB}+\mathrm{SN}+\mathrm{NRT}$. The study protocol was approved by Western Institutional Review Board.

\section{Recruitment and Enrollment}

Participants were new registered users of BecomeAnEX.org, a free, publicly available smoking cessation Web site. Eligibility criteria were current smoking, age 18 years or older, and U.S. residence. 
Exclusion criteria included contraindications to NRT (pregnant or breastfeeding, recent cardiac problems, and current NRT use). Enrollment was automated using a Web-based clinical trials management system. Immediately following registration on BecomeAnEX, participants were shown an invitation that briefly described the study. Interested participants completed online eligibility screening followed by informed consent. Upon completion of a Web-based baseline survey, participants were randomized to treatment, stratified by gender and baseline motivation to quit.

\section{Interventions}

Smoking Cessation Web Site (WEB)

Participants had full access to the BecomeAnEX Web site which was developed in collaboration with Mayo Clinic Nicotine Dependence Center, ${ }^{37}$ in accordance with the U.S. Public Health Service Clinical Practice Guidelines. ${ }^{1}$ The site helps users develop problem-solving and coping skills, educates users about effective cessation medications, and delivers social support as part of treatment. Educational content focuses on preparing for quit day, coping with slips, and preventing relapse, and a series of videos explain nicotine addiction and how medication can help. BecomeAnEX guides and supports smokers through the following interactive components: a series of Addiction Videos which provides information on pharmacotherapy; a Quit Date tool that assists users in selecting a quit date; a Build Your Support System exercise to identify helpful supporters; a Beat Your Smoking Triggers exercise to identify strategies to dissociate cigarettes from triggers; a Cigarette Tracker exercise to identify smoking triggers; a Choose a Quit Smoking Aid exercise, in which users indicate their plans for pharmacotherapy use; and Community, a large online network of current and former smokers who communicate via private messages and public posts in blogs, groups, forums, and on member profile pages.

\section{Social Network Intervention}

Participants received proactive communications from three established members of BecomeAnEX ("Integrators") who were longstanding, very active members of the community. Selection and recruitment of these Integrators has been described elsewhere. ${ }^{36}$ The overarching goal of these communications was to facilitate the integration of new members into the BecomeAnEX online social network. Within 24 hours of study enrollment, the Integrators posted a public message on a new member's personal BecomeAnEX profile page ("wall") to welcome them, encourage them to fill out their profile, or comment on some aspect of their profile. Integrators did not receive any formal training in cessation treatment and were instructed not to address questions or comments specifically about cessation other than to encourage participants' efforts and direct them to relevant content and tools on the Web site.

\section{Nicotine Replacement Therapy Intervention}

Participants were mailed a free 4-week supply of the NRT product of their choice (patch, gum, or lozenge) within 3 days of randomization. NRT was provided as an over-the-counter product to parallel the experience subjects would have if they purchased NRT on their own. To facilitate accurate reporting of product use at the follow-up assessment, a printed calendar was included with the NRT shipment for participants to record product use.

\section{Data Collection}

Data on treatment adherence were obtained through three sources: automated tracking data of general utilization metrics gathered through Omniture/Adobe Analytics software, ${ }^{38}$ use of specific Web site components stored in unified event logs, and self-report followup data. All metrics were extracted at 3 months postrandomization since this is typically the period during which Web site utilization is highest. ${ }^{39-41}$ Omniture/Adobe Analytics is a Web analytics tool that is used to monitor BecomeAnEX. It allows for reliable capture of general Web site utilization metrics. Unified event logs track user behavior across platforms (ie, Web site or mobile site) and store the source of the action and the date/time of its occurrence. Self-report data were gathered via mixed-mode follow-up (online surveys, telephone for online nonresponders, and mailed survey for those unreachable by telephone). Mailed reminders sent prior to each follow-up and monetary incentives for survey completion were used to maximize follow-up rates. Automated tracking data were available for all participants; self-report data were available only for those reached at 3 months.

\section{Measures}

\section{General Web Site Utilization Metrics}

Number of return visits to the Web site following registration, total number of pages viewed, and number of minutes spent using the site were examined as general Web site utilization metrics.

\section{Use of Skills Training Components}

The total number of page views related to use of skills training content and interactive exercises was extracted from BecomeAnEX event logs. In addition, data on use/nonuse of the following components were extracted: (1) Addiction and Medication Videos, (2) Quit Date tool, (3) Build Your Support System, (4) Beat Your Smoking Triggers, (5) Cigarette Tracker, and (6) Choose Your Quit Smoking Aid.

\section{Use of Social Support}

The following metrics of engagement in the BecomeAnEX Community were examined as metrics of intratreatment social support: whether the participant visited the Community, total number of Community page views, whether they made a wall post, whether they viewed member profiles, whether they wrote a blog, whether they read others' blogs, whether they commented on a blog, and whether they sent private messages.

\section{Use of Pharmacotherapy}

At 3 months, participants were asked about their use of medication. Participants were first queried about medication provided by the study (receipt of NRT from the study, use of study-provided NRT, and the number of days study-provided NRT was used). Next, participants reported whether they had obtained any cessation medication on their own. Those who had were subsequently asked about use of NRT (patch, gum, lozenge, spray, inhaler), Zyban/bupropion, and Chantix/varenicline.

\section{Statistical Analyses}

The aim of this study was to evaluate the comparative effectiveness of $\mathrm{WEB}+\mathrm{SN}$, WEB+NRT, and $\mathrm{WEB}+\mathrm{SN}+\mathrm{NRT}$ for increasing treatment adherence versus $\mathrm{WEB}$ alone at 3 months postrandomization. Treatment adherence was operationalized as general Web site utilization metrics, use of skills training components on the Web site, intratreatment social support via the community, and pharmacotherapy use. Our primary hypothesis was that all three intervention groups would outperform the control group for all outcomes 
of interest. This hypothesis was tested by first conducting an omnibus test of any between-group differences using chi-square statistics for categorical variables and Kruskal-Wallis one-way analysis of variance for continuous variables. Significant omnibus differences were followed by examination of pairwise contrasts for each intervention group against the control group, using chi-square tests for categorical variables and Wilcoxon two-sample rank-sum tests for continuous variables. Our secondary hypothesis was that the combined intervention $\mathrm{WEB}+\mathrm{SN}+\mathrm{NRT}$ would have a greater impact on the outcomes of interest than either one of the single intervention arms, WEB+SN or WEB+NRT. Of note, this hypothesis was also nested within the hypothesis of any between-group differences and was only evaluated if the omnibus test was significant. To control family-wise type I error at alpha $=0.05$ across all five comparisons of interest, we used a significance threshold of alpha $=0.01$ for these comparisons.

\section{Results}

The study CONSORT diagram has been published elsewhere. ${ }^{42}$ Briefly, 20670 individuals were screened, 11022 were eligible $(53 \%)$, and 5290 (48\% eligible) were randomized to the final sample. Reasons for ineligibility were as follows: 1043 not current smokers, 1095 under 18 years of age, and 7510 with contraindication to NRTs. Baseline characteristics of the sample have been previously reported and approximate the characteristics of nationally representative samples of treatment-seeking smokers. ${ }^{42}$ The enrolled sample was $60.8 \%$ female, $80.1 \%$ White, $94.4 \%$ non-Hispanic, and $72.6 \%$ with at least some college education. Mean age was 41.8 years $(S D=13.2)$ and $93.5 \%$ reported daily internet use. Participants were daily smokers $(96.2 \%)$ who smoked an average of 16.6 cigarettes per day $(S D=8.5)$, and the majority $(83.0 \%)$ planned to quit in the next 30 days. There were no significant differences on any baseline characteristics between treatment arms. The overall follow-up rate at 3 months was $62.3 \%$ : by treatment arm, rates were $58.4 \%$ (WEB), $56.3 \%$ (WEB+SN), 68.7\% (WEB+NRT), and $66.0 \%$ (WEB+SN+NRT). A higher percentage of participants were reached in the two NRT arms than in the other two arms $(67.3 \%$ vs. $57.4 \%$ respectively, $p<.001$ ).

Treatment arm differences in general Web site utilization metrics (visits and time), skills training tools, and use of intratreatment social support are summarized in Tables 1 and 2 for the overall randomized sample $(N=5290)$. In addition, the use of smoking cessation medications and other cessation treatments is summarized in Tables 3 and 4 (respectively) for the overall 3-month responder sample $(N=3297)$. Omnibus $p$ values are shown in the rightmost column. Pairwise differences of the three intervention arms against the control arm are highlighted using the letters a/A if they are significant at the two-sided $\alpha=0.01$ and $\alpha=0.001$ levels, respectively. Pairwise differences of single intervention arms (WEB+SN, WEB+NRT) against the combined intervention arm (WEB+SN+NRT) are highlighted using the letters $\mathrm{b} / \mathrm{B}$ if they are significant at the two-sided $\alpha=0.01$ and $\alpha=0.001$ levels, respectively.

\section{Treatment Effects on General Web Site Utilization}

Omnibus testing suggested that study arms differed in terms of all three Web site utilization metrics listed in Table 1 (return site visits, total page views, and time on site). Examination of pairwise contrasts revealed that all three intervention arms outperformed the control arm on all metrics $(p<.01)$. Additionally, the combined intervention arm outperformed both of the single intervention arms across all metrics $(p<.001)$. As a result, study findings provided support for both hypotheses of interest in terms of increasing general metrics of Web site utilization.

\section{Treatment Effects on Use of Skills Training Components}

Intervention effects were also seen in terms of promoting the use of skills training components on BecomeAnEX. Only WEB+SN+NRT outperformed WEB in the use of all seven components listed in Table $1(p<.001)$, whereas WEB+NRT outperformed WEB in four of these components: skills training page views, watching Addiction Videos, using the Quit Date tool, and using the Build Your Support System tool. WEB+SN had the smallest impact on the use of skills training components, with statistically significant improvements compared to WEB limited to a $5 \%$ increase in the proportion of study participants watching Addiction Videos. As for differences between the combined and single intervention arms, WEB+SN+NRT outperformed $\mathrm{WEB}+\mathrm{SN}$ in all aspects of skills training, other than the watching of Addiction Videos. No differences were observed between WEB+SN+NRT and WEB+NRT in use of any of the skills training components.

\section{Treatment Effects on Use of Intratreatment Social Support}

Omnibus testing indicated that the four study arms differed across all eight metrics of community engagement listed in Table 2. Pairwise contrasts revealed that the WEB+SN+NRT arm outperformed the control arm on all metrics of community engagement. In particular, it was the only intervention arm to have significantly promoted use of private messages and community page views among community users. Both $\mathrm{WEB}+\mathrm{SN}$ and $\mathrm{WEB}+\mathrm{NRT}$ increased the proportion of users who visited the community and made a wall post compared to WEB. WEB+SN additionally promoted profile views and blog comments, whereas WEB+NRT promoted the reading of blogs. As for differences between the combined and single intervention arms, $\mathrm{WEB}+\mathrm{SN}+\mathrm{NRT}$ outperformed WEB+NRT in all aspects of community engagement, other than community page views among visitors to the community. It also outperformed $\mathrm{WEB}+\mathrm{SN}$ in terms the proportion of users who visited the community, made a wall post, and made or read a blog post.

\section{Treatment Effects on Smoking Cessation Medication Use}

Omnibus testing revealed the presence of significant between-group differences in the use of any cessation medication (ie, considering both study and non-study) at 3 months (Table 3). Significant pairwise differences indicated higher medication use in both study arms that received NRT compared to the control arm ( $p$ values $<.001$ ), while $\mathrm{WEB}+\mathrm{SN}$ did not increase the use of cessation medication over WEB. With regard to non-study medication, use was higher in $\mathrm{WEB}+\mathrm{SN}$ compared to $\mathrm{WEB}+\mathrm{SN}+\mathrm{NRT}(p<.01)$, but the two arms in which study NRT was provided did not differ from each other.

\section{Treatment Effects on Use of Other Cessation Treatments}

Although provided in Table 4 for completeness, no differences were detected between study arms in the use of other quit methods. Omnibus tests failed to attain even nominal levels of statistical 

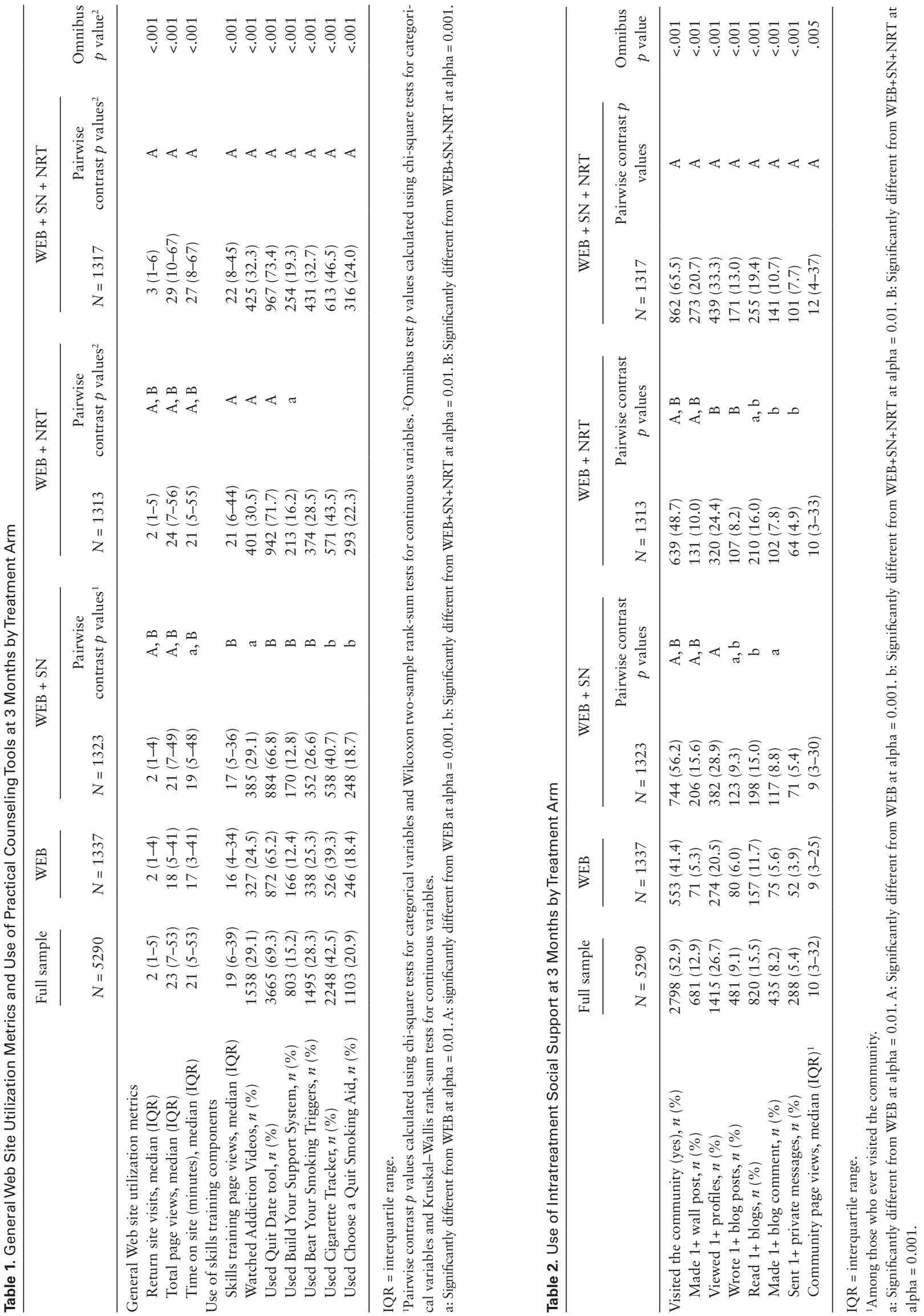
significance. As a result, we did not examine the significance of any pairwise contrasts. The quit methods used most commonly among participants were e-cigarettes $(37.1 \%)$, switching brands/cutting back $(35.8 \%)$, and pamphlet/book $(16.3 \%)$.

\section{Discussion}

Adherence to Web-based smoking cessation interventions-and to Internet health behavior change programs more broadly-is a welldocumented challenge. ${ }^{17-19,27,31,40,41,43}$ Less well understood are what strategies are effective in increasing adherence or engagement to digital interventions. This trial examined the impact of two intervention strategies on adherence to the core components of cessation treatment in an online intervention. The SN intervention was very effective in increasing overall Web site utilization and both active and passive engagement in the community, but did not yield significant increases in the use of skills training components or cessation medication. The NRT intervention was very effective in increasing overall Web site utilization, the use of some of the skills training components, community engagement, and NRT uptake. The combined intervention yielded the strongest effects across all metrics of treatment adherence.

To our knowledge, this is the first study to attempt to influence the formation of social ties in an online network using some of its existing members. These "Integrators" served as a "welcome wagon" to expose new study participants to the nature of the community and to encourage them to browse. This approach is increasingly common in online community management, ${ }^{44-47}$ but has not previously been tested experimentally. Proactive outreach from Integrators in this study resulted in significant increases in treatment intensity across all three Web site utilization metrics (return visits, page views, and time on site). This outreach also triggered a significant increase in the proportion of participants who visited the community in the single intervention arm. Slightly over half of participants randomized to that arm visited the community at least once, a necessary precursor to any additional support or influence that may be gleaned from ongoing community engagement. The strongest effects of the social network intervention on community engagement were observed on profile views and wall posts. Given that the intervention itself was delivered via wall posts, these increases serve as a fidelity check that participants were aware of and responded to the intervention. It is also noteworthy that the intervention increased the proportion of study participants who participated actively in the community by posting a blog or commenting on others' blog posts. While the overall proportion of individuals who participated in this manner remained below $10 \%$, active communication among only a minority of members is typical of most online networks. ${ }^{48}$ In addition to increasing individual-level treatment adherence, the social network intervention represents a scalable and promising approach that may help ensure the health of an online social network by maintaining a steady volume of user-generated content. Other studies have documented the characteristics of online social network "super-users", ${ }^{24,49,50}$ to our knowledge, this is the first study to demonstrate the effectiveness of a social network approach that draws on super-users to increase initial levels of engagement in an online community for smoking cessation.

It is noteworthy that the free course of NRT increased the use of skills training. Unlike the SN intervention, NRT increased the percentage of users who used the Quit Date tool, suggesting that NRT may have triggered a greater proportion of users to make a quit attempt. Increased use of skills training components may have been driven by the higher rate of quit attempts as participants sought information 
Table 4. Use of Other Quit Methods at 3 Months by Treatment Arm

\begin{tabular}{|c|c|c|c|c|c|c|}
\hline & Full sample & WEB & $\mathrm{WEB}+\mathrm{SN}$ & WEB + NRT & $\mathrm{WEB}+\mathrm{SN}+\mathrm{NRT}$ & \\
\hline & $N=3297$ & $N=781$ & $N=745$ & $N=902$ & $N=869$ & Omnibus $p$ value $^{a}$ \\
\hline Pamphlet/book, $n(\%)$ & $465(16.3)$ & $130(19.1)$ & $106(16.6)$ & $117(14.9)$ & $112(15.0)$ & .111 \\
\hline Individual counseling, $n(\%)$ & $67(2.4)$ & $15(2.2)$ & $12(1.9)$ & $23(2.9)$ & $17(2.3)$ & .599 \\
\hline Clinic or group counseling, $n(\%)$ & $62(2.2)$ & $12(1.8)$ & $16(2.5)$ & $21(2.7)$ & $13(1.7)$ & .484 \\
\hline Phone counseling, $n(\%)$ & $143(5.0)$ & $41(6.0)$ & $37(5.8)$ & $38(4.9)$ & $27(3.6)$ & .146 \\
\hline Digital intervention (Web/mobile), $n(\%)$ & $220(7.7)$ & $59(8.7)$ & $45(7.0)$ & $66(8.4)$ & $50(6.7)$ & .401 \\
\hline E-cigarettes, $n(\%)$ & $1060(37.1)$ & $242(35.6)$ & $243(38.0)$ & $307(39.2)$ & $266(35.6)$ & .391 \\
\hline Switching to chew tobacco/snuff, $n(\%)$ & $58(2.0)$ & $13(1.9)$ & $15(2.3)$ & $15(1.9)$ & $15(2.0)$ & .936 \\
\hline Switching brands or cut back, $n(\%)$ & $1020(35.8)$ & $240(35.3)$ & $235(36.7)$ & $289(36.9)$ & $257(34.4)$ & .722 \\
\hline Acupuncture, $n(\%)$ & $24(0.8)$ & $5(0.7)$ & $6(0.9)$ & $7(0.9)$ & $6(0.8)$ & .978 \\
\hline Hypnosis, $n(\%)$ & $63(2.2)$ & $16(2.4)$ & $16(2.5)$ & $15(1.9)$ & $16(2.1)$ & .885 \\
\hline Alternative methods, $n(\%)$ & $209(7.3)$ & $50(7.4)$ & $47(7.3)$ & $57(7.3)$ & $55(7.4)$ & .999 \\
\hline
\end{tabular}

The table only includes data from participants who completed the 3-month follow-up assessment.

a Significance of pairwise contrasts was not assessed, as none of the omnibus tests attained significance at the alpha $=0.05$ level.

about preparing to quit and using NRT. We also saw a boost in community engagement above WEB alone, but to a lesser extent than the $\mathrm{SN}$ intervention. Higher levels of community engagement in the WEB+NRT arm may have been partially driven by information seeking from peers about NRT. To our knowledge, this is the first study to observe increases in skills training and social support as a result of providing free NRT. Not surprisingly, there was a dramatic boost in the use of medication in the WEB+NRT and WEB+SN+NRT arms. Only about one-third of participants in WEB and WEB+SN used any cessation medication, which is consistent with national surveys. ${ }^{51,52}$ In contrast, $84 \%$ of participants in the WEB+NRT and WEB+SN+NRT arms used any cessation medication. These findings highlight the fact that provision of NRT through a Web-based system-whether as part of a study or integrated into a population-based cessation programcan substantially decrease logistic barriers. ${ }^{53}$ We hypothesized that the social network intervention would increase medication adoption based on communication of social norms. The fact that reported cessation medication use rates of $28.7 \%$ (WEB) and $30.6 \%$ (WEB+SN) were not significantly different may be related to community-specific norms around medication, or to the medication views of the Integrators themselves. Understanding the nature of medication-related communications that participants were exposed to is an important area for further research before concluding that a social network approach is not effective in increasing adherence to pharmacotherapy.

Several limitations of this study should be noted. First, the followup rates ranged from $56 \%$ to $69 \%$ across arms, suggestive of differential attrition. While these follow-up rates are higher than those achieved in other Internet cessation trials, ${ }^{5,6}$ our ability to assess the use of medication and other quit methods was affected by loss to follow-up. Study attrition did not impact assessment of general Web site utilization, use of skills training components, and community use where we have complete tracking data on all users. Second, this study did not specifically examine intratreatment social ties through formal social network analyses. Thus, we are not able to discern whether the increase in treatment adherence in the social network arms is a result of ties formed exclusively with study Integrators or the extent to which the $\mathrm{SN}$ intervention resulted in the formation of ties with other members of the social network. Formal social network analyses in conjunction with an examination of utilization metrics will provide important insights to address this question. Finally, although the interventions tested yielded statistically significant increases in treatment utilization, the practical significance may be considered more modest. It is important to note that brief engagement with an Internet program may not necessarily indicate failure: Some participants may disengage after successful cessation or finding the information they desired. ${ }^{54}$ Given the intractable nature of digital engagement, we are encouraged by these findings.

Despite these limitations, this study has important implications for implementation science generally and the study of Internetbased behavior change interventions in particular. Several reviews have called for more research on the factors that influence use of proven treatments. ${ }^{14,55}$ This study demonstrated the effectiveness of two approaches for improving adherence to the evidence-based components of smoking cessation treatment in the context of a Web-based intervention. The social network approach used in this trial is commonly seen in online communities, is highly scalable, and could be easily implemented by lay members of an existing community. NRT is often part of population-based cessation programs $^{56}$ and can be delivered easily as part of either telephonic or Web-based interventions. Its delivery in this study mimicked realworld conditions, providing promising evidence for the benefit of pharmacotherapy on the uptake and use of other cessation treatment components. Taken together, the positive effects observed for the single and combined intervention arms on general and feature-specific metrics of treatment adherence provide an important foundation for future research to optimize Web-based cessation interventions.

\section{Funding}

This research was funded by the National Cancer Institute (1R01CA15548901A1 to ALG).

\section{Declaration of Interests}

ALG, SC, MSA, RSN, and DBA are employees of Truth Initiative, a nonprofit public health foundation that runs BecomeAnEX.org. NKC is an employee of MYH Inc, a digital health and wellness company that provides smoking cessation services.

\section{Acknowledgments}

Study concept and design: ALG, GDP, NKC, RS, and DBA.

Obtained funding: ALG.

Acquisition of data: ALG and SC. 
Analysis and interpretation of data: ALG, GDP, SC, BE, and MSA. Drafting of the manuscript: ALG, GDP, SC, and MSA.

Review of manuscript: BE, NKC, RSN, and DBA.

Trial Registration: Clinicaltrials.gov NCT01544153.

\section{References}

1. Fiore M, Jaén C, Baker T; Tobacco Use and Dependence Guideline Panel. Treating Tobacco Use and Dependence: 2008 Update. Clinical Practice Guideline. Rockville, MD: U.S. Department of Health and Human Services, Public Health Service; 2008.

2. Graham AL, Chang Y, Fang Y, et al. Cost-effectiveness of internet and telephone treatment for smoking cessation: an economic evaluation of The iQUITT Study. Tob Control. 2013;22(6):e11.

3. Smit ES, Evers SM, de Vries H, Hoving C. Cost-effectiveness and costutility of Internet-based computer tailoring for smoking cessation. J Med Internet Res. 2013;15(3):e57.

4. Stanczyk NE, Smit ES, Schulz DN, et al. An economic evaluation of a video- and text-based computer-tailored intervention for smoking cessation: a cost-effectiveness and cost-utility analysis of a randomized controlled trial. PLoS One. 2014;9(10):e110117.

5. Graham AL, Carpenter KM, Cha S, et al. Systematic review and metaanalysis of Internet interventions for smoking cessation among adults. Subst Abuse Rehabil. 2016;7:55-69.

6. Civljak M, Sheikh A, Stead LF, Car J. Internet-based interventions for smoking cessation. Cochrane Database Syst Rev. 2013(7).

7. Walters ST, Wright JA, Shegog R. A review of computer and Internet-based interventions for smoking behavior. Addict Behav. 2006;31(2):264-277.

8. Myung S-K, McDonnell DD, Kazinets G, Seo HG, Moskowitz JM. Effects of Web-and computer-based smoking cessation programs: meta-analysis of randomized controlled trials. Arch Int Med. 2009;169(10):929-937.

9. Graham AL, Papandonatos GD, Cobb CO, et al. Internet and Telephone Treatment for smoking cessation: mediators and moderators of short-term abstinence. Nicotine Tob Res. 2015;17(3):299-308.

10. McClure JB, Peterson D, Derry H, et al. Exploring the "active ingredients" of an online smoking intervention: a randomized factorial trial. Nicotine Tob Res. 2014.

11. Strecher VJ, Shiffman S, West R. Moderators and mediators of a webbased computer-tailored smoking cessation program among nicotine patch users. Nicotine Tob Res. 2006;8(suppl 1):S95-S101.

12. Strecher VJ, McClure J, Alexander G, et al. The role of engagement in a tailored web-based smoking cessation program: randomized controlled trial. J Med Internet Res. 2008;10(5):5-5.

13. Brownson RC, Fielding JE, Maylahn CM. Evidence-based public health: a fundamental concept for public health practice. Annu Rev Public Health. 2009;30:175-201.

14. Nilsen P. Making sense of implementation theories, models and frameworks. Implement Sci. 2015;10:53.

15. Mabry PL, Olster DH, Morgan GD, Abrams DB. Interdisciplinarity and systems science to improve population health: a view from the NIH Office of Behavioral and Social Sciences Research. Am J Prev Med. 2008;35(2 suppl):S211-S224.

16. Cobb NK, Graham AL, Byron MJ, Niaura RS, Abrams DB, Workshop Participants online social networks and smoking cessation: a scientific research agenda. J Med Internet Res. 2011;13(4):e119.

17. Danaher BG, Boles SM, Akers L, Gordon JS, Severson HH. Defining participant exposure measures in Web-based health behavior change programs. J Med Internet Res. 2006;8(3):e15.

18. Danaher BG, Seeley JR. Methodological issues in research on web-based behavioral interventions. Ann Behav Med. 2009;38(1):28-39.

19. Donkin L, Christensen H, Naismith SL, Neal B, Hickie IB, Glozier N. A systematic review of the impact of adherence on the effectiveness of e-therapies. J Med Internet Res. 2011;13(3):e52.

20. Pike KJ, Rabius V, McAlister A, Geiger A. American Cancer Society's QuitLink: randomized trial of Internet assistance. Nicotine Tob Res. 2007;9(3):415-420.
21. Rabius V, Pike KJ, Wiatrek D, McAlister AL. Comparing internet assistance for smoking cessation: 13-month follow-up of a six-arm randomized controlled trial. J Med Internet Res. 2008;10(5):e45.

22. Saul JE, Schillo BA, Evered S, et al. Impact of a statewide Internet-based tobacco cessation intervention. J Med Internet Res. 2007;9(3):e28.

23. Japuntich SJ, Zehner ME, Smith SS, et al. Smoking cessation via the internet: a randomized clinical trial of an internet intervention as adjuvant treatment in a smoking cessation intervention. Nicotine Tob Res. 2006;8(suppl 1):S59-S67.

24. Cobb NK, Graham AL, Bock BC, Papandonatos G, Abrams DB. Initial evaluation of a real-world Internet smoking cessation system. Nicotine Tob Res. 2005;7(2):207-216.

25. Balmford J, Borland R, Benda P. Patterns of use of an automated interactive personalized coaching program for smoking cessation. J Med Internet Res. 2008;10(5):e54.

26. McClure JB, Shortreed SM, Bogart A, et al. The effect of program design on engagement with an internet-based smoking intervention: randomized factorial trial. J Med Internet Res. 2013;15(3):e69.

27. Alkhaldi G, Hamilton FL, Lau R, Webster R, Michie S, Murray E. The effectiveness of prompts to promote engagement with digital interventions: a systematic review. J Med Internet Res. 2016;18(1):e6.

28. Poirier J, Cobb NK. Social influence as a driver of engagement in a webbased health intervention. J Med Internet Res. 2012;14(1):e36.

29. Thorpe KE, Zwarenstein M, Oxman AD, et al. A pragmatic-explanatory continuum indicator summary (PRECIS): a tool to help trial designers. CMAJ. 2009;180(10):E47-E57.

30. Bandura A. Social Foundations of Thought and Action: A Social Cognitive Theory. Englewood Cliffs, NJ: Prentice-Hall; 1986.

31. Cugelman B, Thelwall M, Dawes P. Online interventions for social marketing health behavior change campaigns: a meta-analysis of psychological architectures and adherence factors. J Med Internet Res. 2011;13(1):e17.

32. Cobb NK, Mays D, Graham AL. Sentiment analysis to determine the impact of online messages on smokers' choices to use varenicline. J Natl Cancer Inst Monogr. 2013;2013(47):224-230.

33. Miller N, Frieden TR, Liu SY, et al. Effectiveness of a large-scale distribution programme of free nicotine patches: a prospective evaluation. Lancet. 2005;365(9474):1849-1854.

34. van Boven JF, Vemer P. Higher adherence during reimbursement of pharmacological smoking cessation treatments. Nicotine Tob Res. 2016;18(1):56-63.

35. Glasgow RE, Green LW, Klesges LM, et al. External validity: we need to do more. Ann Behav Med. 2006;31(2):105-108.

36. Graham AL, Cha S, Papandonatos GD, et al. Improving adherence to webbased cessation programs: a randomized controlled trial study protocol. Trials. 2013;14:48.

37. McCausland KL, Curry LE, Mushro A, Carothers S, Xiao H, Vallone DM. Promoting a web-based smoking cessation intervention: implications for practice. Cases Public Health Commun Mark. 2011;5:3-26.

38. Adobe Analyst. 2015. www.adobe.com/marketing-cloud/web-analytics/ marketing-analytics-capabilities.html.

39. Cobb CO, Graham AL. Use of non-assigned interventions in a randomized trial of internet and telephone treatment for smoking cessation. Nicotine Tob Res. 2014;16(10):1289-1297.

40. Eysenbach G. The law of attrition. J Med Internet Res. 2005;7(1):e11.

41. Eysenbach G. The law of attrition revisited - Author's reply. J Med Internet Res. 2006;8(3):e21.

42. Cha S, Erar B, Niaura RS, Graham AL. Baseline characteristics and generalizability of participants in an internet smoking cessation randomized trial. Ann Behav Med. 2016;50(5):751-761.

43. Brouwer W, Kroeze W, Crutzen R, et al. Which intervention characteristics are related to more exposure to internet-delivered healthy lifestyle promotion interventions? A systematic review. J Med Internet Res. 2011;13(1):e2.

44. Williams RL, Cothrel J. Four smart ways to run online communities. Sloan Manage Rev. 2000(Summer):81-91.

45. Ng D. Online Community Management For Dummies. Hoboken, NJ: John Wiley \& Sons; 2011. 
46. Brown C. Developing structure to welcome new members in your communities. My Community Manager.

47. Millington R. Buzzing Communities: How to Build Bigger, Better, and More Active Online Communities. Lexington, KY: FeverBee; 2012.

48. Nielsen J. The 90-9-1 Rule for Participation Inequality in Social Media and Online Communities. 2006. www.webcitation.org/6VmCdF1C3. Accessed December 2, 2014.

49. van Mierlo T, Voci S, Lee S, Fournier R, Selby P. Superusers in social networks for smoking cessation: analysis of demographic characteristics and posting behavior from the Canadian Cancer Society's smokers' helpline online and StopSmokingCenter.net. J Med Internet Res. 2012;14(3):e66.

50. Cobb NK, Graham AL, Abrams DB. Social network structure of a large online community for smoking cessation. Am J Public Health. 2010;100(7):1282-1289.
51. Cokkinides VE, Ward E, Jemal A, Thun MJ. Under-use of smoking-cessation treatments: results from the National Health Interview Survey, 2000. Am J Prev Med. 2005;28(1):119-122.

52. Shiffman S, Brockwell SE, Pillitteri JL, Gitchell JG. Use of smoking-cessation treatments in the United States. Am J Prev Med. 2008;34(2):102-111.

53. Koh HK, Sebelius KG. Promoting prevention through the Affordable Care Act. N Engl J Med. 2010;363(14):1296-1299.

54. Saul JE, Amato MS, Cha S, Graham AL. Engagement and attrition in Internet smoking cessation interventions: Insights from a cross-sectional survey of "one-hit-wonders". Internet Interv . 2016;5:23-29.

55. Rabin BA, Glasgow RE, Kerner JF, Klump MP, Brownson RC. Dissemination and implementation research on community-based cancer prevention: a systematic review. Am J Prev Med. 2010;38(4):443-456.

56. North American Quitline Consortium. Free and Discounted Cessation Medication. 2016; http://map.naquitline.org/reports/medication/.Accessed August 11, 2016. 